\title{
PERTURBATION OF A GLOBALLY STABLE STEADY STATE
}

\author{
H. L. SMITH AND P. WALTMAN \\ (Communicated by Linda Keen)
}

\begin{abstract}
It is shown that within a parameterized family of semi-dynamical systems enjoying a mild uniform dissipative condition, the property that a locally asymptotically stable steady state is globally attracting is an open condition in the parameters.
\end{abstract}

\section{INTRODUCTION}

The ultimate goal in the analysis of most dynamical systems arising in applications is to determine the asymptotic behavior of its solutions. In the language of dynamical systems, one wishes to determine the nature of the global attractor. In doing this, one often uses special properties of functions in the equations or, indeed, of the nature of the model itself. A particular example of the latter is in several models of microbial competition based on the chemostat. In the chemostat, two (or more) organisms compete for a limiting nutrient. Thus the model has features common to a predator-prey model in the consumption of the nutrient. However, the basic conservation property of the chemostat allows a reduction of the order to two competitive equations where the theory of monotone dynamical systems is applicable. See the monograph [8] for many examples. Monotone dynamical systems have a strong tendency to convergence [7]. In many of these chemostat-like systems, the result is that the global attractor (of the interior) is a rest point (also called an equilibrium or steady state). However, a slight change in the model parameters destroys the form of the conservation principle and the reduction to a monotone dynamical system is no longer possible. Similarly, a Liapunov function may be known under certain restrictive conditions but not for the general system.

In section 2 we provide perturbation theorems for both discrete and continuous dynamical systems that will address this problem. The local perturbation of an asymptotically stable rest point is a standard result (see [6] for upper semicontinuity of the basin of attraction); the focus here is retaining the rest point as a globally stable attractor. Essentially, we show that within a parameterized family of dynamical systems enjoying a mild uniform dissipative condition, the property that a locally asymptotically stable steady state is globally attracting is an open condition in the parameters.

Received by the editors May 20, 1997.

1991 Mathematics Subject Classification. Primary 34C35, 34E10, 58F30.

The first author was supported by NSF Grant DMS 9300974, and the second author was supported by NSF Grant DMS 9424592 and an award from the University Research Council of Emory University.

(C)1999 American Mathematical Society 
In section 3, our main result is applied to the Droop model of phytoplankton growth in a chemostat environment. If the phytoplankton death rate is ignored, solutions are attracted to a lower dimensional manifold and the system becomes tractable; inclusion of a death rate makes this property less explicit but our result applies.

\section{Perturbation theorems}

Let $T: U \times \Lambda \rightarrow U$ be continuous where $U \subset X, X$ is a Banach space, and $\Lambda$ is a metric space with metric $\rho$. We sometimes write $T_{\lambda}(x)=T(x, \lambda)$ and use the notation $B_{X}(x, s)\left(B_{\Lambda}(\lambda, s)\right)$ for the open ball of radius $s$ about the point $x \in X$ $(\lambda \in \Lambda)$. For a linear operator $A$ on $X$, we write $r(A)$ for its spectral radius.

Theorem 2.1. Let $\left(x_{0}, \lambda_{0}\right) \in U \times \Lambda, B_{X}\left(x_{0}, \delta\right) \subset U$ for some $\delta>0$ and assume that $D_{x} T(x, \lambda)$ exists and is continuous in $B_{X}\left(x_{0}, \delta\right) \times \Lambda$. Suppose that $T\left(x_{0}, \lambda_{0}\right)=$ $x_{0}, r\left(D_{x} T\left(x_{0}, \lambda_{0}\right)\right)<1$, and $T_{\lambda_{0}}^{n} x \rightarrow x_{0}$ for every $x \in U$. In addition, suppose that:

(H1) For each $\lambda \in \Lambda$, there is a set $B_{\lambda} \subset U$ such that for each $x \in U$, there exists $N=N(x, \lambda)$ such that $T_{\lambda}^{N} x \in B_{\lambda}$.

(H2) $C \equiv \overline{\bigcup_{\lambda \in \Lambda} T_{\lambda}\left(B_{\lambda}\right)}$ is compact in $U$.

Then there exists $\epsilon_{0}>0$ and a continuous map $\hat{x}: B_{\Lambda}\left(\lambda_{0}, \epsilon_{0}\right) \rightarrow U$ such that $\hat{x}\left(\lambda_{0}\right)=x_{0}, T(\hat{x}(\lambda), \lambda)=\hat{x}(\lambda)$ and:

$$
T_{\lambda}^{n} x \rightarrow \hat{x}(\lambda), \quad x \in U, \lambda \in B_{\Lambda}\left(\lambda_{0}, \epsilon_{0}\right) .
$$

Proof. We may suppose that the norm on $X$ is such that $\left\|D_{x} T\left(x_{0}, \lambda_{0}\right)\right\|<\rho<1$. See ([10], page 795 or [5], sec. 2.5.2). As $D_{x} T(x, \lambda)$ is continuous, there exist $\epsilon_{1}, \eta>0$ such that $\left\|D_{x} T(x, \lambda)\right\|<\rho$ for $x \in \overline{B_{X}\left(x_{0}, \eta\right)}$ and $\lambda \in B_{\Lambda}\left(\lambda_{0}, \epsilon_{1}\right)$. Choose $\epsilon_{0}<\epsilon_{1}$ such that $\left\|T\left(x_{0}, \lambda_{0}\right)-T\left(x_{0}, \lambda\right)\right\|<(1-\rho) \eta$ for $\lambda \in B_{\Lambda}\left(\lambda_{0}, \epsilon_{0}\right)$. Then, for $x, x^{\prime} \in \overline{B_{X}\left(x_{0}, \eta\right)}$ and $\lambda \in B_{\Lambda}\left(\lambda_{0}, \epsilon_{0}\right)$, we have

$$
\left\|T(x, \lambda)-T\left(x^{\prime}, \lambda\right)\right\| \leq \int_{0}^{1}\left\|D_{x} T\left(s x+(1-s) x^{\prime}, \lambda\right)\right\| d s\left\|x-x^{\prime}\right\| \leq \rho\left\|x-x^{\prime}\right\|
$$

and

$$
\begin{aligned}
\left\|T(x, \lambda)-x_{0}\right\| & \leq\left\|T(x, \lambda)-T\left(x_{0}, \lambda\right)\right\|+\left\|T\left(x_{0}, \lambda\right)-T\left(x_{0}, \lambda_{0}\right)\right\| \\
& <\rho\left\|x-x_{0}\right\|+(1-\rho) \eta \leq \eta .
\end{aligned}
$$

Thus, $T_{\lambda}$ is a uniform contraction self-mapping of $\overline{B_{X}\left(x_{0}, \eta\right)}$ for $\lambda \in B_{\Lambda}\left(\lambda_{0}, \epsilon_{0}\right)$. The uniform contraction mapping theorem implies the existence of the continuous map $\hat{x}: B_{\Lambda}\left(\lambda_{0}, \epsilon_{0}\right) \rightarrow \overline{B_{X}\left(x_{0}, \eta\right)}$ such that $T(\hat{x}(\lambda), \lambda)=\hat{x}(\lambda)$ and $\hat{x}\left(\lambda_{0}\right)=x_{0}$. Furthermore, $T_{\lambda}^{m} x \rightarrow \hat{x}(\lambda)$ for every $x \in \overline{B_{X}\left(x_{0}, \eta\right)}$ and $\lambda \in B_{\Lambda}\left(\lambda_{0}, \epsilon_{0}\right)$.

By choosing $\epsilon_{0}$ smaller, if necessary, we claim that for $\lambda \in B_{\Lambda}\left(\lambda_{0}, \epsilon_{0}\right)$ and $x \in C$, $T_{\lambda}^{m} x \in B\left(x_{0}, \eta\right)$ for some $m$. If not, there exist $\lambda_{n} \in \Lambda, \lambda_{n} \rightarrow \lambda_{0}$, and $x_{n} \in C$ such that $\left\|T_{\lambda_{n}}^{m} x_{n}-x_{0}\right\|$ gen for all $m \geq 0, n \geq 1$. As $C$ is compact, we may assume that $x_{n} \rightarrow x \in C$. But $x_{0}$ is globally attracting for $T_{\lambda_{0}}$ so there is a $p$ such that $\left\|T_{\lambda_{0}}^{p} x-x_{0}\right\|<\eta / 2$. Hence, by continuity of the function $F(x, \lambda) \equiv T_{\lambda}^{p} x$, $F\left(x_{n}, \lambda_{n}\right) \rightarrow F\left(x, \lambda_{0}\right)=T_{\lambda_{0}}^{p} x$ and therefore $\left\|T_{\lambda_{n}}^{p} x_{n}-x_{0}\right\|<\eta$ for all large $n$, a contradiction. The claim is established.

Now, given $x \in U$ and $\lambda \in B_{\Lambda}\left(\lambda_{0}, \epsilon_{0}\right)$, there exists $N$ such that $T_{\lambda}^{N} x \in B_{\lambda}$ so $T_{\lambda}^{N+1} x \in C$. By the previous paragraph, $T_{\lambda}^{m} x \in B_{X}\left(x_{0}, \eta\right)$ for some, and hence all large, $m$. Obviously, $T_{\lambda}^{m} x \rightarrow \hat{x}(\lambda)$. 
The main assertion of Theorem 2.1 is (2.1). The existence of the perturbed fixed point $\hat{x}(\lambda)$ is usually not an issue, indeed, it may be known explicitly. Continuity of eigenvalues under perturbation implies the local stability of $\hat{x}(\lambda)$. We have given conditions for the global asymptotic stability of the fixed point $x_{0}$ to be stable to perturbation: the perturbed fixed point $\hat{x}(\lambda)$ is also globally attracting.

In the applications, the set $B$ in (H1) will typically be bounded and the compactness of $C$ will derive from a uniform complete continuity assumption on the $\left\{T_{\lambda}\right\}_{\lambda \in \Lambda}$ in case $X$ is infinite dimensional. In finite dimensional spaces $X$, (H2) is implied by (H1) if $B$ is bounded. Typically, the discrete dynamical system will be defined on a larger set, perhaps containing other invariant sets. $U$ may then be chosen to be some positively invariant (for each $T_{\lambda}$ ) subset of the basin of attraction of $x_{0}$.

Remark 2.1. The assumption that $x_{0}$ is an interior point of $U$ is unnecessarily restrictive. An examination of the proof indicates that it is sufficient that $T$ can be extended to $B_{X}\left(x_{0}, \delta\right) \times \Lambda$ for some $\delta>0$ and has a continuous derivative on that set and that $B_{X}\left(x_{0}, \delta\right) \cap U$ is convex. Alternatively, one-sided derivatives with respect to some cone or wedge in $X$ may also be used. See, e.g. [1].

We now formulate a continuous-time version of Theorem 2.1 , beginning with some notation and definitions. Let $T: U \times[0, \infty) \times \Lambda \rightarrow U$ be continuous and define a family of (nonlinear) semi-dynamical systems on $U$ parameterized by $\lambda \in \Lambda$. As above, $U \subset X$, where $X$ is a Banach space and $\Lambda$ is a metric space. We often write $T_{\lambda}^{t} x \equiv T(x, t, \lambda)$. More precisely, the continuous map $\mathrm{T}$ defines a family of semi-dynamical systems on $U$ parameterized by $\lambda \in \Lambda$ provided that for each $\lambda \in \Lambda$ :

(i) $T_{\lambda}^{0}=i d_{X}$.

(ii) $T_{\lambda}^{t} \circ T_{\lambda}^{s}=T_{\lambda}^{t+s}$ for $t, s \geq 0$.

The continuous-time version of Theorem 2.1 reads as follows.

Theorem 2.2. Let $T$ be a family of semi-dynamical systems parameterized by $\Lambda$. Let $\left(x_{0}, \lambda_{0}\right) \in U \times \Lambda, B_{X}\left(x_{0}, \delta\right) \subset U$ for some $\delta>0$ and assume that $D_{x} T(x, t, \lambda)$ exists for $(x, t, \lambda) \in B_{X}\left(x_{0}, \delta\right) \times[0, \infty) \times \Lambda$ and for each fixed $t \geq 0$, $D_{x} T(x, t, \lambda)$ is continuous on $B_{X}\left(x_{0}, \delta\right) \times \Lambda$. Suppose that $T_{\lambda_{0}}^{t} x_{0}=x_{0}$ for all $t \geq 0$, $U(t) \equiv D_{x} T_{\lambda_{0}}^{t}\left(x_{0}\right)$ defines a strongly continuous semigroup with negative growth bound $(r(U(t))=\exp (-\omega t)$ with $\omega>0)$, and $T_{\lambda_{0}}^{t}(x) \rightarrow x_{0}$ for each $x \in U$. In addition, suppose that:

(H1) For each $\lambda \in \Lambda$, there is a subset $B_{\lambda}$ of $U$ such that for each $x \in U, T_{\lambda}^{t}(x) \in$ $B_{\lambda}$ for all large $t$.

(H2) $C \equiv \overline{\bigcup_{\lambda \in \Lambda} T_{\lambda}^{s}\left(B_{\lambda}\right)}$ is compact in $U$ for some $s>0$.

Then there exist $\epsilon_{0}>0$ and a continuous map $\hat{x}: B_{\Lambda}\left(\lambda_{0}, \epsilon_{0}\right) \rightarrow U$ such that $\hat{x}\left(\lambda_{0}\right)=x_{0}, T_{\lambda}^{t} \hat{x}(\lambda)=\hat{x}(\lambda)$ for $t \geq 0$, and

$$
T_{\lambda}^{t} x \rightarrow \hat{x}(\lambda), \quad x \in U, \lambda \in B_{\Lambda}\left(\lambda_{0}, \epsilon_{0}\right) .
$$

Proof. Let $P: U \times \Lambda \rightarrow U$ be defined by $P_{\lambda}(x)=P(x, \lambda)=T_{\lambda}^{s} x$ where $s>0$ is as in (H2). Using that $P_{\lambda}^{n}(x)=T_{\lambda}^{n s} x$, observe that all the hypotheses of Theorem 2.1 hold for $P$. By that theorem, the function $\hat{x}$ exists satisfying $P_{\lambda}(\hat{x}(\lambda))=T_{\lambda}^{s} \hat{x}(\lambda)=$ $\hat{x}(\lambda)$ and $T_{\lambda}^{n s} x \rightarrow \hat{x}(\lambda)$ for every $x \in U$ and $\lambda \in B_{\Lambda}\left(\lambda_{0}, \epsilon_{0}\right)$. For $y \in U$ and $\tau \geq 0$, put $x=T_{\lambda}^{\tau} y \in U$ in the previous limit to obtain $T_{\lambda}^{n s+\tau} y \rightarrow \hat{x}(\lambda)$. Thus, $T_{\lambda}^{\tau}\left(T_{\lambda}^{n s} y\right) \rightarrow \hat{x}(\lambda)$. But $T_{\lambda}^{\tau}\left(T_{\lambda}^{n s} y\right) \rightarrow T_{\lambda}^{\tau} \hat{x}(\lambda)$ so $T_{\lambda}^{\tau} \hat{x}(\lambda)=\hat{x}(\lambda)$ for each $\tau \geq 0$ and $\lambda \in B_{\Lambda}\left(\lambda_{0}, \epsilon_{0}\right)$. 
We claim that $T_{\lambda}^{t} x \rightarrow \hat{x}(\lambda)$ for each $x \in U$ and $\lambda \in B_{\Lambda}\left(\lambda_{0}, \epsilon_{0}\right)$. If not, there exist $x \in U, \lambda \in B_{\Lambda}\left(\lambda_{0}, \epsilon_{0}\right), \eta>0$ and $t_{n} \rightarrow \infty$ such that $\left\|T_{\lambda}^{t_{n}} x-\hat{x}(\lambda)\right\| \geq \eta$ for $n \geq 1$. Write $t_{n}=k_{n} s+\tau_{n}$ where $k_{n}$ is a positive integer, $\tau_{n} \in[0, s)$, and $k_{n} \rightarrow \infty$. We may assume that $\tau_{n} \rightarrow \tau \in[0, s]$. Then

$$
T_{\lambda}^{t_{n}} x=T_{\lambda}^{k_{n} s+\tau_{n}} x=T_{\lambda}^{\tau_{n}}\left(T_{\lambda}^{k_{n} s} x\right) \rightarrow T_{\lambda}^{\tau} \hat{x}(\lambda)=\hat{x}(\lambda),
$$

a contradiction.

As a corollary, we formulate a result for ordinary differential equations in finite dimensions. Consider

$$
x^{\prime}=f(x, \lambda)
$$

where $f: U \times \Lambda \rightarrow \mathbb{R}^{n}$ is continuous, where $U \subset \mathbb{R}^{n}$ and $\Lambda \subset \mathbb{R}^{k}$ and $D_{x} f(x, \lambda)$ is continuous on $U \times \Lambda$. We assume that solutions of initial value problems are unique and remain in $U$ for all $t \geq 0$ and each $\lambda \in \Lambda$. We write $x(t, z, \lambda)$ for the solution of (2.3) satisfying $x(0)=z$.

Corollary 2.3. Assume that $\left(x_{0}, \lambda_{0}\right) \in U \times \Lambda, x_{0} \in \operatorname{Int} U, f\left(x_{0}, \lambda_{0}\right)=0$, all eigenvalues of $D_{x} f\left(x_{0}, \lambda_{0}\right)$ have negative real part, and $x_{0}$ is globally attracting for solutions of (2.3) with $\lambda=\lambda_{0}$. If

(H1) there exists a compact set $D \subset U$ such that for each $\lambda \in \Lambda$ and each $z \in U$, $x(t, z, \lambda) \in D$ for all large $t$,

then there exist $\epsilon>0$ and a unique point $\hat{x}(\lambda) \in U$ for $\lambda \in B_{\Lambda}\left(\lambda_{0}, \epsilon\right)$ such that $f(\hat{x}(\lambda), \lambda)=0$ and $x(t, z, \lambda) \rightarrow \hat{x}(\lambda)$ as $t \rightarrow \infty$ for all $z \in U$.

If the existence and local asymptotic stability of $\hat{x}(\lambda)$ are known to hold for all $\lambda \in \Lambda$, then Theorem 2.1 implies that the subset of $\Lambda$ for which $\hat{x}(\lambda)$ is globally asymptotically stable is an open subset. It may not be all of $\Lambda$ however. A simple example is afforded by $(2.3)$ where $f: \mathbb{R} \times(-1,1) \rightarrow \mathbb{R}$ is defined by $f(x, \lambda)=$ $-x^{3}+2 x^{2}-(1+\lambda) x$. For all $\lambda \in(-1,1)$ the equilibrium $\hat{x}(\lambda)=0$ is locally asymptotically stable but it is globally attracting only when $\lambda \in(0,1)$ because a saddle-node bifurcation occurs at $\lambda=0$ producing a pair of equilibria near $x=1$ for $\lambda<0$.

We remark that similar results can be proved for more general compact invariant sets than single equilibria or fixed points.

\section{An EXAmple}

In this section, we give an application of our results to the Droop model of phytoplankton growth on a limiting nutrient in a chemostat, considered in Chapter 8 of [8]. Although the example is simple, it illustrates the difficulties encountered in applications where more than one steady state is present. Consider the system of ordinary differential equations:

$$
\begin{aligned}
x^{\prime} & =x(\mu(Q)-m), \\
Q^{\prime} & =\rho(S, Q)-\mu(Q) Q, \\
S^{\prime} & =1-S-x \rho(S, Q)
\end{aligned}
$$

where $x$ denotes (scaled) phytoplankton biomass, $Q$ denotes average stored nutrient concentration per unit biomass of phytoplankton, and $S$ is the nutrient concentration in the chemostat. When $m=1$, system (3.1) is just (2.4) in Chapter 8 of [8]. The value $m=1$ results from assuming that the phytoplankton death rate is 
negligible so that the only loss of biomass is due to "washout" at the same rate that nutrient is lost (see the third equation). It is common to ignore the death rate of cells (as in [8]) because it is typically small compared to the washout rate and the system is much simpler when it is ignored, as we will see below. Here, we allow $m$ to differ from $m=1$, although not by much. From a biological perspective, the case $m=1+d$, where $d>0$ represents a death rate for phytoplankton, is of most interest and the restriction that $d<<1$ is not a severe one.

Concerning the functions $\rho$ and $\mu$, it is assumed that they are continuously differentiable and satisfy:

$$
\begin{aligned}
\mu(P) & =0 \\
\mu^{\prime}(Q) & >0, \quad Q \geq P
\end{aligned}
$$

where $P>0$ and

$$
\begin{aligned}
\rho(0, Q) & =0, \\
\frac{\partial \rho}{\partial S} & >0 \\
\frac{\partial \rho}{\partial Q} & \leq 0 .
\end{aligned}
$$

The domain of interest is given by

$$
U=\{(x, Q, S): x>0, Q \geq P, S \geq 0\}
$$

which is easily seen to be positively invariant. There is a unique root $Q^{*}$ of

$$
\rho\left(1, Q^{*}\right)-\mu\left(Q^{*}\right) Q^{*}=0
$$

because of the monotonicity of $\rho$ and $\mu$. We assume that $\mu\left(Q^{*}\right)>1$ and restrict $m$ to the set

$$
\Lambda=\left\{m: m<\mu\left(Q^{*}\right)\right\} .
$$

Note that $m=1$ belongs to $\Lambda$. If $m \in \Lambda$, then there exists a unique steady state for (3.1) in $U$, given by

$$
E_{1}=\left(x_{0}, Q_{0}, S_{0}\right),
$$

where $\mu\left(Q_{0}\right)=m, \rho\left(S_{0}, Q_{0}\right)=m Q_{0}$, and $x_{0}=\frac{1-S_{0}}{m Q_{0}}>0$. The positivity of $x_{0}$, implied by $S_{0}<1$, follows automatically from the monotonicity of $\rho$ and $\mu$ and the definition of $Q^{*}$. It is an easy exercise to show by the Routh-Hurwitz criterion that $E_{1}$ is locally asymptotically stable. In [8], Theorem 2.2 , it is shown that if $m=1$, then $E_{1}$ is globally attracting for (3.1) in $U$. We use Corollary 2.3 to establish the following result.

Theorem 3.1. There exists $\epsilon>0$ such that for $|m-1|<\epsilon, E_{1}$ attracts all solutions of (3.1) with initial data in $U$.

The steady state $E_{0}=\left(0, Q^{*}, 1\right)$ on the boundary of $U$ greatly complicates the proof of the theorem.

To see why the case $m=1$ is special, consider the quantity

$$
T=S+Q x
$$

If $m=1$, it satisfies the differential equality

$$
T^{\prime}=1-S-Q x=1-T .
$$


In this special case, the differential equality implies that all solutions approach the surface $S+Q x=1$ at an exponential rate. This in turn implies that the system can be reduced to a planar system by dropping the $S$ equation and making the substitution $S=1-Q x$ in the remaining two equations. In [8], we refer to this as a conservation law for (3.1). Similar conservation laws are common in the analysis of chemostat models. However, if $m \neq 1$, one has only the differential inequality

$$
\begin{aligned}
T^{\prime} & =1-S-m Q x \\
& \leq 1-\delta T,
\end{aligned}
$$

where $\delta=\min (1, m)$ and a similar reduction to a lower order system is not obvious. One can, however, conclude that the system (3.1) is dissipative. In order to fix $\delta$ independent of $m$, we hereafter restrict $m$ to $m \geq 1 / 2$ and set $\delta=1 / 2$.

Lemma 3.2. $B_{m} \equiv\left[0,(\delta P)^{-1}\right] \times\left[P, Q^{*}\right] \times[0,1]$ attracts all solutions of $(3.1)$.

Proof. The differential inequality above implies $\lim _{\sup _{t \rightarrow \infty}} T(t) \leq \delta^{-1}$. As $Q \geq$ $P>0$, we conclude from this that $\lim \sup _{t \rightarrow \infty} x(t) \leq(\delta P)^{-1}$. The differential inequality $S^{\prime} \leq 1-S$, from the third equation of (3.1), leads to $\lim \sup _{t \rightarrow \infty} S(t) \leq 1$. Thus, for each $\epsilon>0$ and all large $t$, the second equation leads to $Q^{\prime} \leq \rho(1+\epsilon, Q)-$ $\mu(Q) Q$. Thus, $\limsup _{t \rightarrow \infty} Q(t) \leq Q_{\epsilon}^{*}$ where $Q_{\epsilon}^{*}$ is the unique root of the right side. Letting $\epsilon \rightarrow 0$, we find that $\lim \sup _{t \rightarrow \infty} Q(t) \leq Q^{*}$.

Lemma 3.3. There exists $\eta>0$ and $\epsilon>0$ such that if $|m-1|<\epsilon$, then $\liminf \operatorname{in}_{t \rightarrow \infty} x(t)>\eta$.

Proof. The assertion says, essentially, that (3.1) is uniformly persistent, uniformly in $m$ near $m=1$. Thus it should not be surprising that we use tools from persistence theory, e.g. [2], [3], [4], [9]. Proposition 1.2 in [9] shows that, with some compactness, one can prove $\liminf _{t \rightarrow \infty} x(t)>\eta$ provided that one can prove $\lim \sup _{t \rightarrow \infty} x(t)>\epsilon$, for some $\epsilon>0$. This holds for a given parameter value $m$ but we want this to hold uniformly in the parameter $m$, for $m$ near $m=1$. If we incorporate the parameter into our dynamical system in the trivial way (e.g. add the equation $m^{\prime}=0$ to system (3.1), we can get this uniformity with respect to $m$ for free (but we need to include $m$ in the compactness condition $\left(C_{1.1}\right)$ of [9]). The compactness requirement is satisfied by Lemma 3.2.

Thus, it suffices to show that the conclusion of the lemma holds with lim sup instead of lim inf. If the latter fails to hold, then there exist $m_{n} \rightarrow 1$ and corresponding solutions $\left(x_{n}, Q_{n}, S_{n}\right)$ of (3.1) with $m=m_{n}$ such that $\limsup _{t \rightarrow \infty} x_{n}(t) \rightarrow 0$. We may assume, after shifting the start time forward if necessary, that $\sup _{t \geq 0} x_{n}(t)$ $\rightarrow 0$. This, together with Lemma 3.2, implies that $S_{n}^{\prime} \geq 1-S_{n}-\eta_{n}$, where $\eta_{n} \rightarrow 0$. Again, after shifting the start time forward if necessary, we can assume that $S_{n}(t) \geq 1-2 \eta_{n}$ for all $t \geq 0$. Therefore, $Q_{n}^{\prime} \geq \rho\left(1-2 \eta_{n}, Q_{n}\right)-\mu\left(Q_{n}\right) Q_{n}$, which implies that $Q_{n}(t) \geq Q^{*}-r_{n}$ for all large $t$, where $Q^{*}-r_{n}$ is the unique root of the right side of the differential inequality and $r_{n} \rightarrow 0$ from continuity $\left(\eta_{n} \rightarrow 0\right)$. By shifting the start time forward, we assume that $Q_{n}(t) \geq Q^{*}-r_{n}$ for all $t \geq 0$. As $\mu\left(Q^{*}\right)-1>0, \mu\left(Q^{*}-r_{n}\right)-m_{n}>0$ for all large $n$ and, since $x_{n}^{\prime} \geq x_{n}\left(\mu\left(Q^{*}-r_{n}\right)-m_{n}\right)$, we conclude that $x_{n}(t) \rightarrow \infty$ as $t \rightarrow \infty$ for large $n$, a contradiction that proves our assertion.

Proof of Theorem 3.1. Lemma 3.2 and Lemma 3.3 imply that we can take for the compact set $D$ in Corollary 2.3 the set $D=\left[\eta / 2,(\delta P)^{-1}+1\right] \times\left[P, Q^{*}+1\right] \times[0,2]$. $E_{1}$ is global attracting for $m=1$. Corollary 2.3 completes the proof. 


\section{REFERENCES}

[1] Amann, H. (1976) Fixed Point equations and nonlinear eigenvalue problems in ordered Banach Spaces, SIAM Rev. 18, 620-709. MR 57:7269

[2] Butler, G. and Waltman, P. (1986) Persistence in dynamical systems, J. Diff. Eqns. 63, 255263. MR 87k:54058

[3] Butler, G., Freedman, H. and Waltman, P. (1986) Uniformly persistent systems, Proc. Amer. Math. Soc. 96, 425-430. MR 87d:58119

[4] Hale, J. and Waltman, P. (1989) Persistence in infinite dimensional systems, SIAM J. Math. Anal. 20, 388-395. MR 90b:58156

[5] Krasnosels'kii, M.A. (1964) Positive solutions of Operator Equations, P. Noordhoff, Groningen, the Netherlands. MR 31:6107

[6] Smith, H.L. (1982) On the basin of attraction of a perturbed attractor, Nonlinear Analysis, 6, 911-917. MR 84i:58077

[7] Smith, H.L. (1995), Monotone Dynamical Systems: An introduction to the Theory of Competitive and Cooperative Systems AMS Math. Surv.\& Monographs, 41, Providence, R.I. MR 96c:34002

[8] Smith, H. and Waltman, P. (1995) The Theory of the Chemostat, Cambridge Univ. Press. MR 96e:92016

[9] Thieme, H. R. (1993) Persistence under relaxed point-dissipativity (with application to an epidemic model), SIAM J. Math. Anal. 24, 407-435. MR 94a:34055

[10] Zeidler, E. (1986) Nonlinear Functional Analysis and its Applications, Springer-Verlag, New York. MR 87f: 47083

Department of Mathematics, Arizona State University, Tempe, Arizona 85287-1804

E-mail address: halsmith@asu.edu

Department of Mathematics and Computer Science, Emory University, Atlanta, Georgia 30322

E-mail address: waltman@mathcs.emory.edu 